\title{
Temporal trends in results of 9170 myocardial perfusion imaging studies (2004 to 2013)
}

\author{
Maseeh uz Zaman1, 2, Nosheen Fatima ${ }^{1,3}$, Unaiza Zaman4, Areeba Zaman4, S. Zahed Rasheed¹, Dad J. Baloch¹ \\ ${ }^{1}$ Nuclear Cardiology Department, Karachi Institute of Heart Diseases (KIHD), Karachi, Pakistan \\ 2Department of Radiology, Aga Khan University Hospital (AKUH), Karachi, Pakistan \\ ${ }^{3}$ Department of Nuclear Medicine, Ziauddin Medical University, Karachi, Pakistan \\ ${ }^{4}$ Students of MBBS, Dow University of Health Sciences (DUHS), Karachi, Pakistan \\ Funding source and disclosure: None
}

[Received 29 IV 2014; Accepted 19 XII 2014]

\begin{abstract}
BACKGROUND: To assess the frequency of normal and abnormal myocardial perfusion imaging (MPI) in a consecutive cohort of patients from Pakistan over a period of 8.5 years.

MATERIAL AND METHODS: We assessed 9170 patients who had undergone stress-rest MPI between January 2004 and June 2013. Patients were assessed for change in demographics, risk factors, and frequency of abnormal and normal MPI.

RESULTS: Overall mean age and male predominance of studied cohort was $\approx 55$ years and $\approx 55: 45$ (M:F), respectively, with no appreciable decline or rise. Marked decline in exercise as mode of stress (from $71 \%$ to $35 \%$, $p$ value significant) was noted during the study period. Regarding the risk factors for CAD, only hypertension was noted to have a significant rising trend during the study period. Trend of MPI results over study period was found non-significant from 2004 till 2006 but from 2007 onward (except 2008), a marginal but significant decline in abnormal MPls (from $45 \%$ to $42 \%$; significant $p$ value) and rise in normal MPI (from $55 \%$ to $58 \%$; significant $p$ value) was noted.

CONCLUSIONS: We conclude that over the past 8.5 years, a marginal but significant decline in abnormal and a rise in normal MPIs trend have been observed. An exorbitant rise in use of vasodilator as a method of stress was also observed. We envisaged a follow-up study to ascertain lower negative predictive value of vasodilator as a possible reason and till than results of this and other such studies must be read cautiously.
\end{abstract}

KEY words: myocardial perfusion imaging, temporal trends, negative predictive value, normal MPI, abnormal MPI

Nuclear Med Rev 2015; 18, 1: 19-24

\section{Background}

Myocardial perfusion imaging (MPI) using ECG-gated single photon emission computerized tomography (GSPECT) since its introduction in late 1980s has become the most commonly performed non-invasive functional cardiac imaging modality. This is attributed as it provides enormous information for risk assessment of patients with known or suspected coronary artery disease and aids in the assessment of myocardial viability [1]. According to 2010 statistic published by American Heart Association (AHA), there has been a progressive decline in cardiac deaths and myocardial infarction,

Correspondence to: Maseeh uz Zaman MD, FASNC, FRCP (Edin) MBBS, MS, FCPS, FEBNM (EU), DCBNC (USA)

Section of Nuclear Medicine, Department of Radiology

Aga Khan University Hospital (AKUH), Karachi, Pakistan

E-mail: maseeh.uzzaman@aku.edu yet the burden of disease remain high [2]. A recently published single center study from USA has documented a declining trend of abnormal GSPECT over a period of two decades [3]. However, despite the high death rates due to non-communicable diseases, by 2010 the leading cause of death in the developing countries including Pakistan would be cardiac deaths [4]. With this diversified pattern of cardiac mortalities, the purpose of this study was to assess the frequency of normal and abnormal MPIs in a consecutive cohort of patients from Pakistan over a period of 8.5 years.

\section{Material and methods}

This study included 10563 consecutive patients who had GSPECT studies at Karachi Institute of Radiotherapy and Nuclear Medicine [KIRAN] (3905 patients from January 2004 till November 2009) and Karachi Institute of Heart Diseases [KIHD] (6658 patients from December 2009 till June 2013). We excluded 1393 
patients with history of valvular disease and cardiomyopathies, while remaining 9170 patients constituted the studied cohort. The study was duly approved by ethical review committee of Institutes. As per departmental practice, pertinent information regarding demographics, presenting complaints, risk factors, and history of intervention were recorded. Dynamic exercise (Bruce or Modified Bruce Protocol) was used in 4469 patients (48.74\%) and remaining 4701 (51.26\%) patients had pharmacological stress using dipyridamole (with or without low level exercise). SPECT MPI was acquired using one day stress and rest or stress only without gating (3905 patients from January 2004 till November 2009) and with gating (6658 patients from December 2009 till June 2013) using 16 frames for post-stress studies under dual head gamma cameras (CardioMD, Philips, Netherland and Mediso, Hungry till November 2009 and only CardioMD, Philips, Netherland from December 2009 onward). Technetium-99m labelled Methoxy IsoBbutyl Isonitrile (Tc-99m MIBI) in dose of $10-15 \mathrm{mCi}(370-555 \mathrm{MBq})$ for stress and $20-30 \mathrm{mCi}$ (740-1110 MBq) for resting studies. Radiotracer was injected at least 1 minute before terminating the treadmill stress test and $3 \mathrm{mi}$ nutes after dipyridamole infusion $(0.142 \mathrm{mg} / \mathrm{kg} / \mathrm{min}$ for 4 minute). We did not use attenuation correction in either study. Left ventricular function parameters like ejection fraction (LVEF in \%), end-diastolic volume (EDV in $\mathrm{ml}$ ) and end-systolic volume (ESV in $\mathrm{ml}$ ) were measured using commercially available software (Autoquan ${ }^{\circledR}$ ). All patients were asked to come with 3-4 hour fasting, stop beta and calcium blocker 24 hours prior, long acting nitrate and tea/coffee at least 12 hour prior the test. All scans were reported by two board certified nuclear cardiologists with $>05$ years' experience.

\section{Statistical analysis}

Data was analyzed by using the MedCalc statistical software version 11.3.10 and SPSS software version 10. Comparisons between patient groups were performed using Student's t test for continuous variables and the $\chi^{2}$ test for categorical variables. Continuous variables were described by mean \pm standard deviation (SD). Odd ratios with 95\% confidence intervals (Cls) were calculated. Statistical significance was defined as $p<0.05$.

\section{Results}

The overall mean age and male predominance of studied cohort over the last 8.5 years was $\approx 55$ year and $\approx 55: 45(M: F)$ respectively with no appreciable decline or rise. Obesity (BMI $>27)$ was found in 1957 (21.03\%) of studied cohort with a significant rise during the study period. A significant decline in exercise as mode of stress was noted during the study period. Regarding the risk factors for $C A D$, only hypertension was noted to have a significant rising trend during the study period, while rest of risk factors did not show any significant shift. Incidence of CAD did also show a significant declining trend during the study period (Table 1, Figure 1). Regarding the trends of MPI results over study period, it was found non-significant from 2004 till 2006 but from 2007 onward (except 2008), a marginal but significant decline in abnormal MPIs was noted (Table 1 and Figure 2).

Comparing the patients' cohort with normal and abnormal MPI each study year, patients with abnormal MPIs were found to be significantly older with male predominance and lower BMI

Table 1. Patients' characteristics

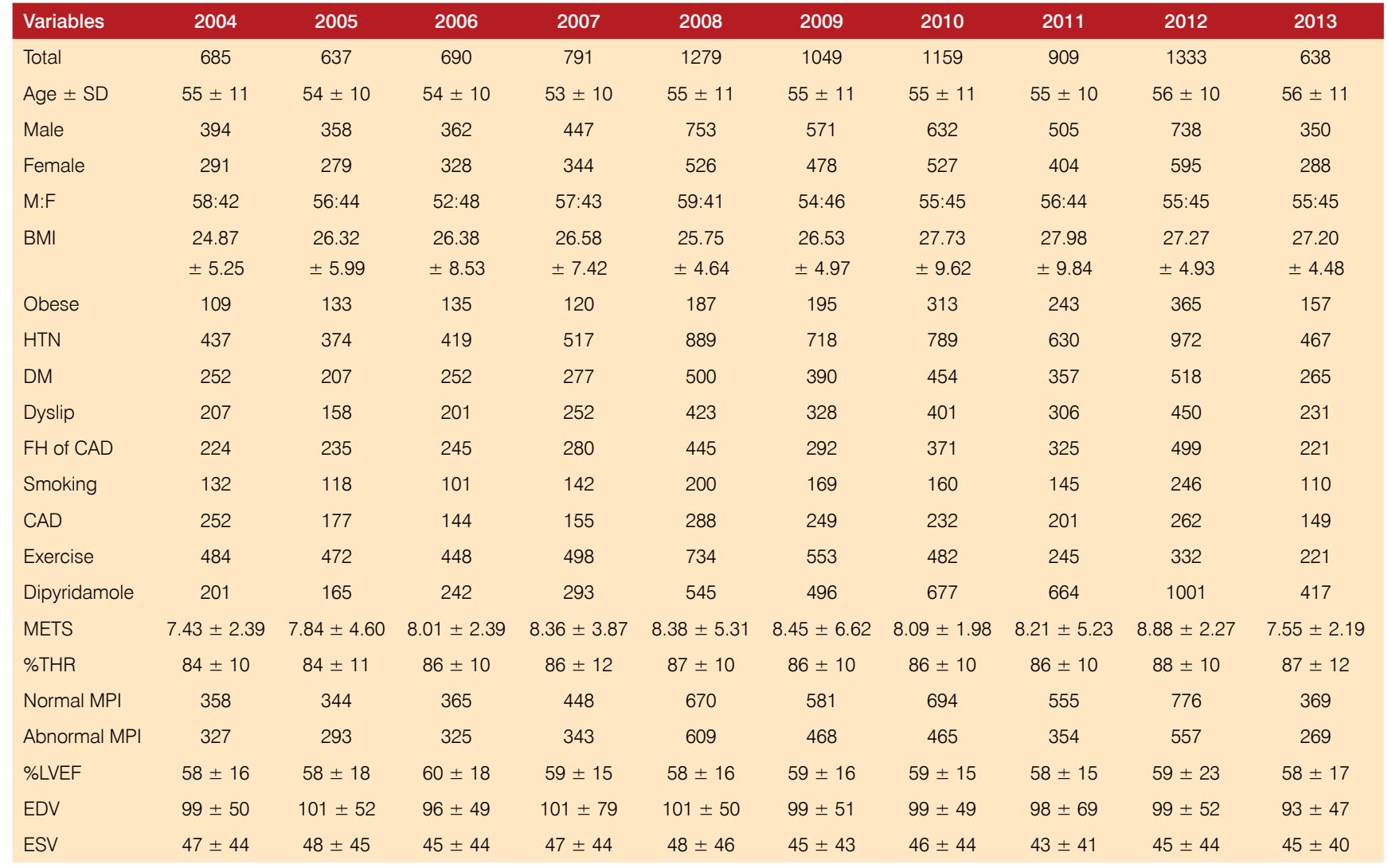




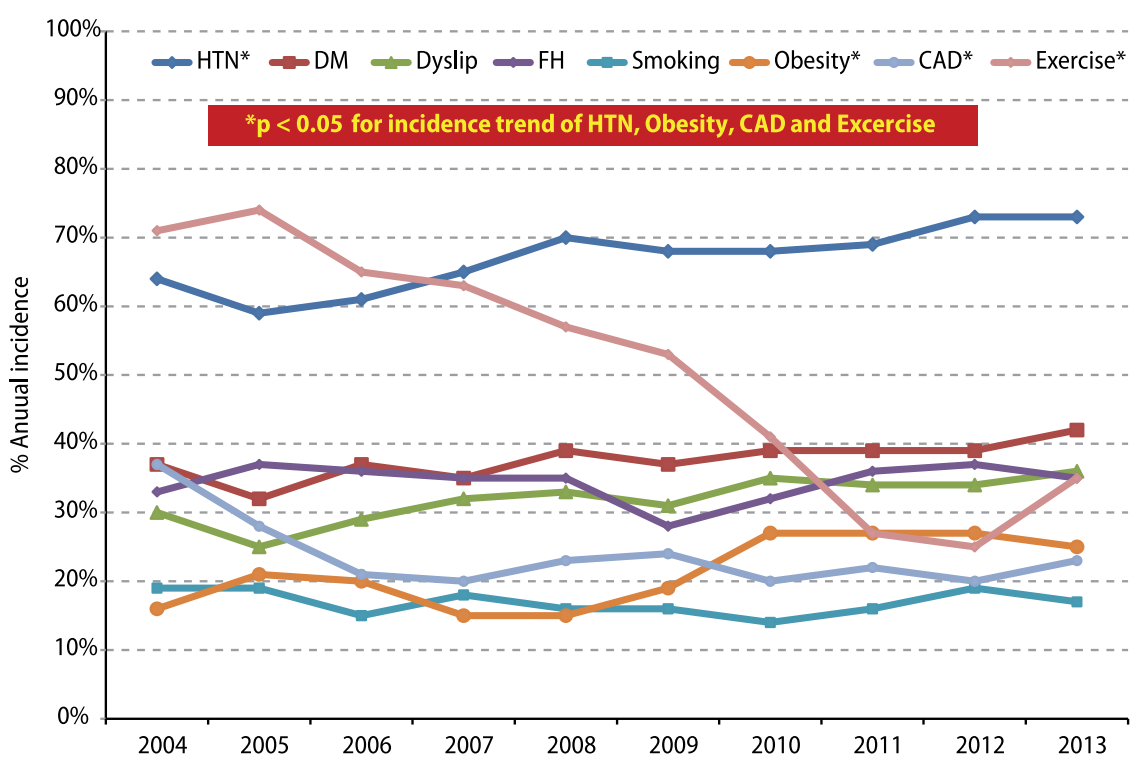

Figure 1. Demographical overall annual incidence trend (\%) from 2004 to 2013

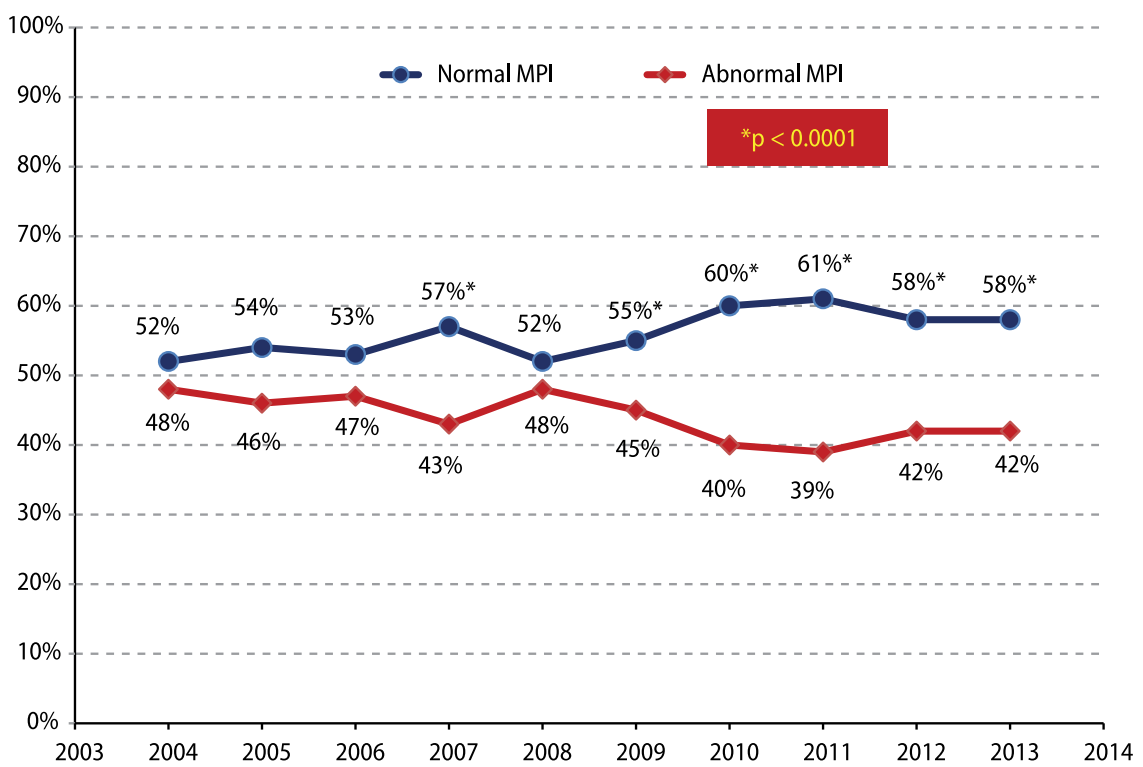

Figure 2. Annual incidence trend (\%) for normal and abnormal myocardial perfusion imaging (2004 to 2013)

(except in 2004 and 2007). Regarding the risk factors, patients with abnormal MPIs were found to have significantly higher prevalence of diabetes and smoking but significant lower prevalence of hypertension (except in 2006). A non-significant prevalence was found for dyslipidemia (except 2005) and family history (except 2004 and 2010) between patients with normal and abnormal MPIs in each study year (Table 2). In patients with normal MPIs, exercise as mode of stress shows a significant declining trend, while hypertension, diabetes, dyslipidemia, family history, smoking, obesity and CAD did not show any significant change in trend over the study period (Table 2 and Figure 3). In patients with abnormal MPIs, a significant declining trend was noted for exercise as mode of stress and presence of $\mathrm{CAD}$, significantly rising trend was observed for diabetes and hypertension, while no significant change in trend was noted for rest of attributes (Table 2 and Figure 4). We have also estimated odds ratio for predicting an abnormal MPI in studied period, and significant odd ratios were estimated for male gender, smoking and CAD for abnormal and normal MPI (Table 3).

\section{Discussion}

In this study we have observed a mild but significant declining trend of abnormal MPIs in the second half of study period. There was a concomitant significant increase in mean BMI of all participants, which is considered to have a positive correlation with CAD and associated mortality [5]. However, there are published studies revealing low incidence of abnormal MPIs in obese patients [6] and plausible explanation for this protective role of higher BMI is reverse epidemiology and obesity paradox [7]. We have also observed an overall significant declining trend of exercise as a mode 


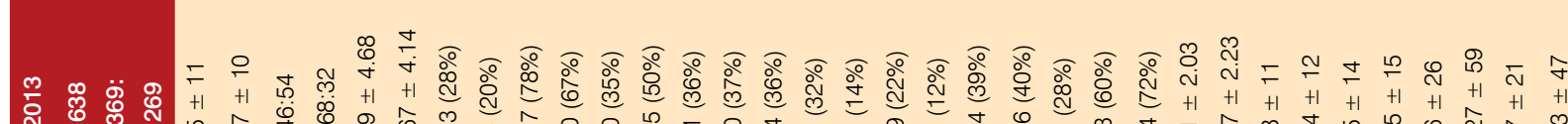

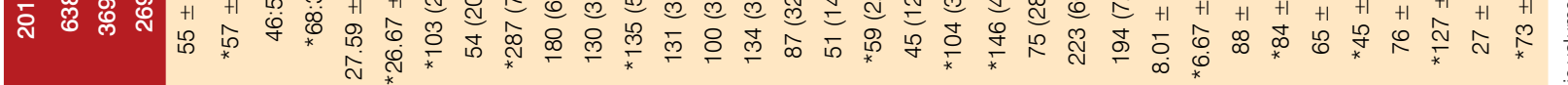

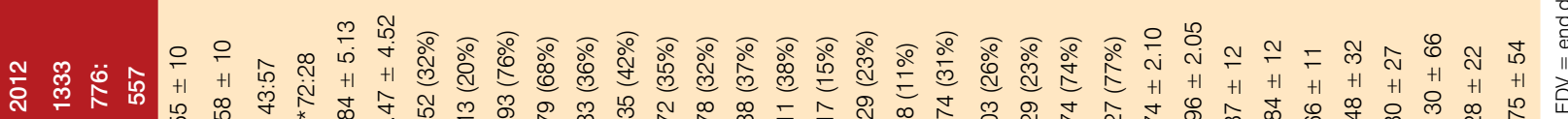

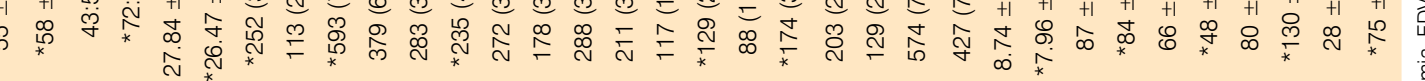

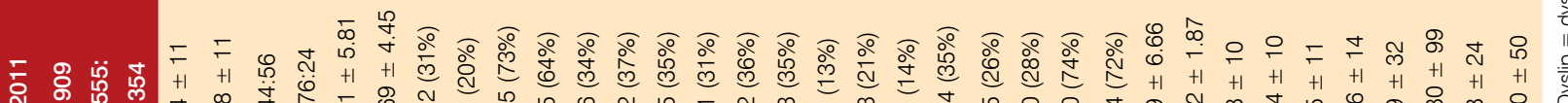

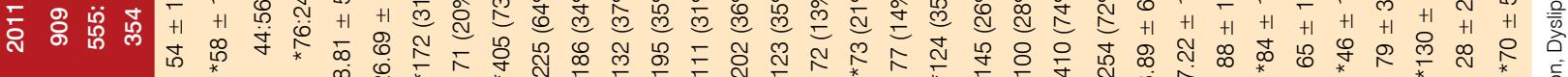

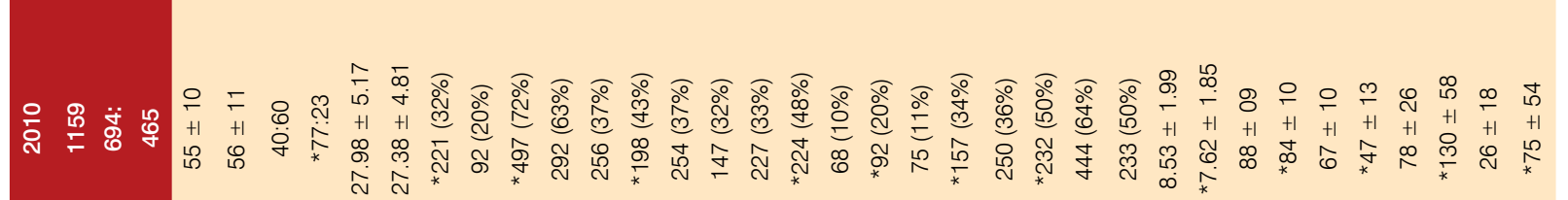

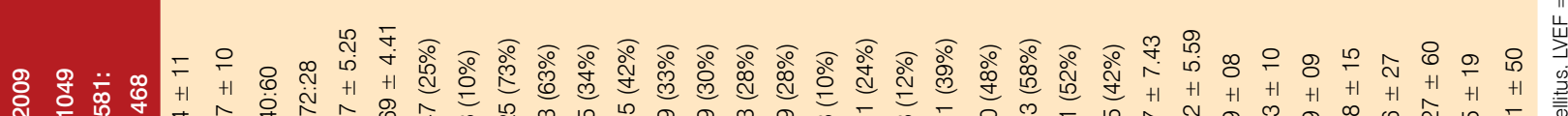
等

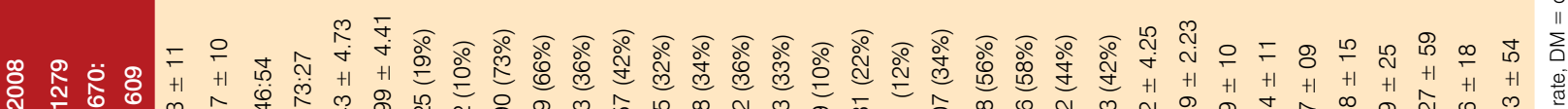

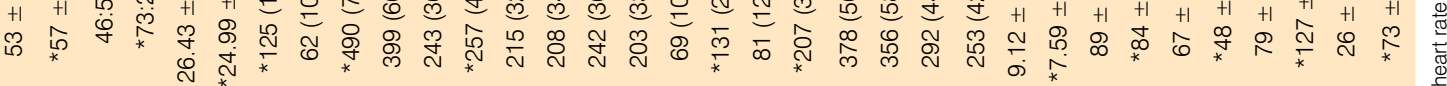

윰

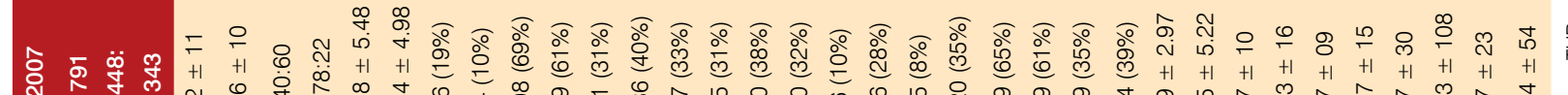

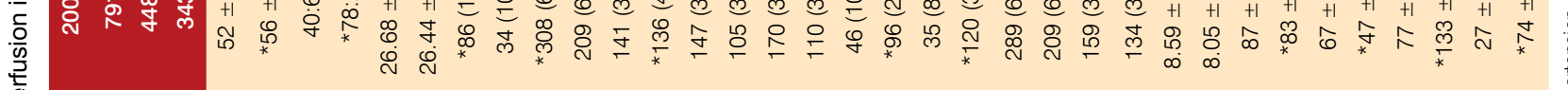

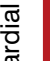

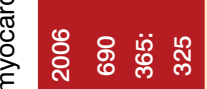

$\frac{\mathrm{c}}{\mathrm{\sigma}}$

$\frac{\pi}{\frac{\pi}{c}}$

ले

$\sum_{\mathbb{\Phi}}^{\stackrel{D}{\Phi}}$

$\stackrel{\frac{0}{n}}{\frac{0}{0}}$

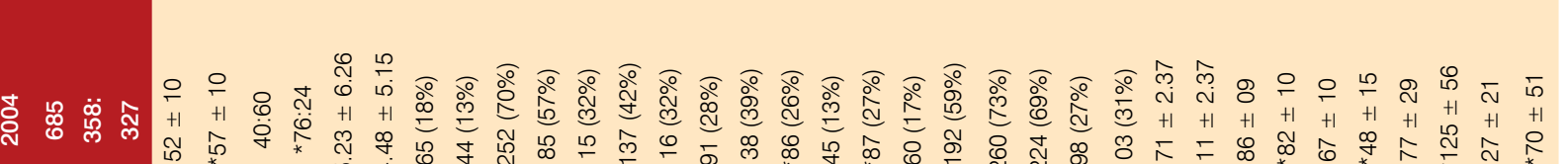

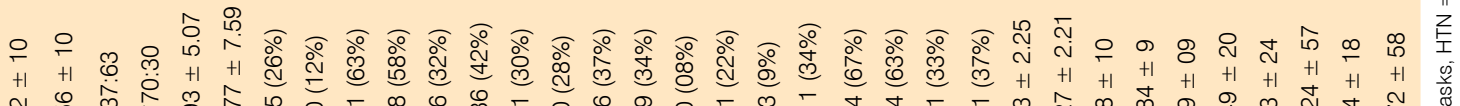

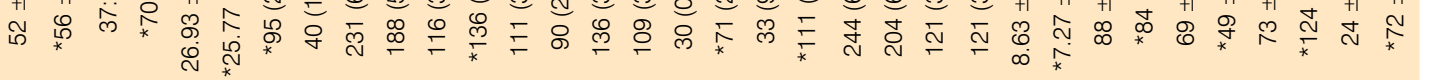

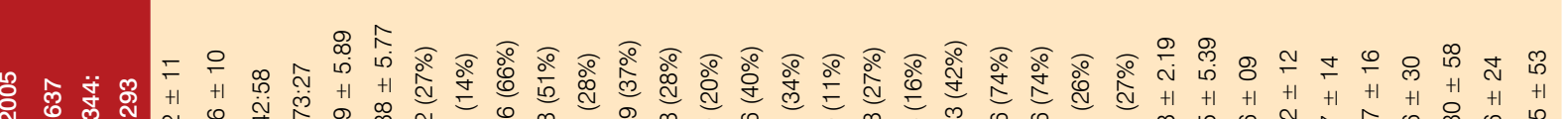

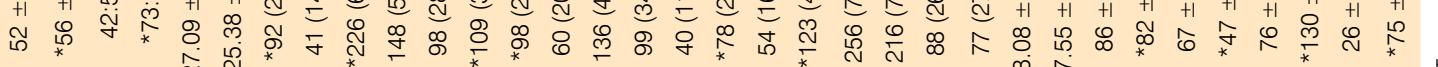

\section{列}

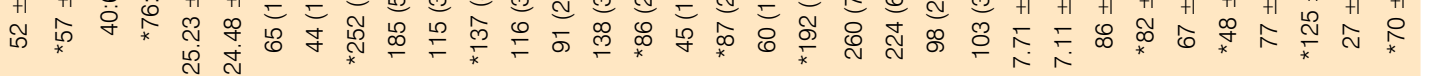

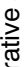

ㅎํำ

촗 


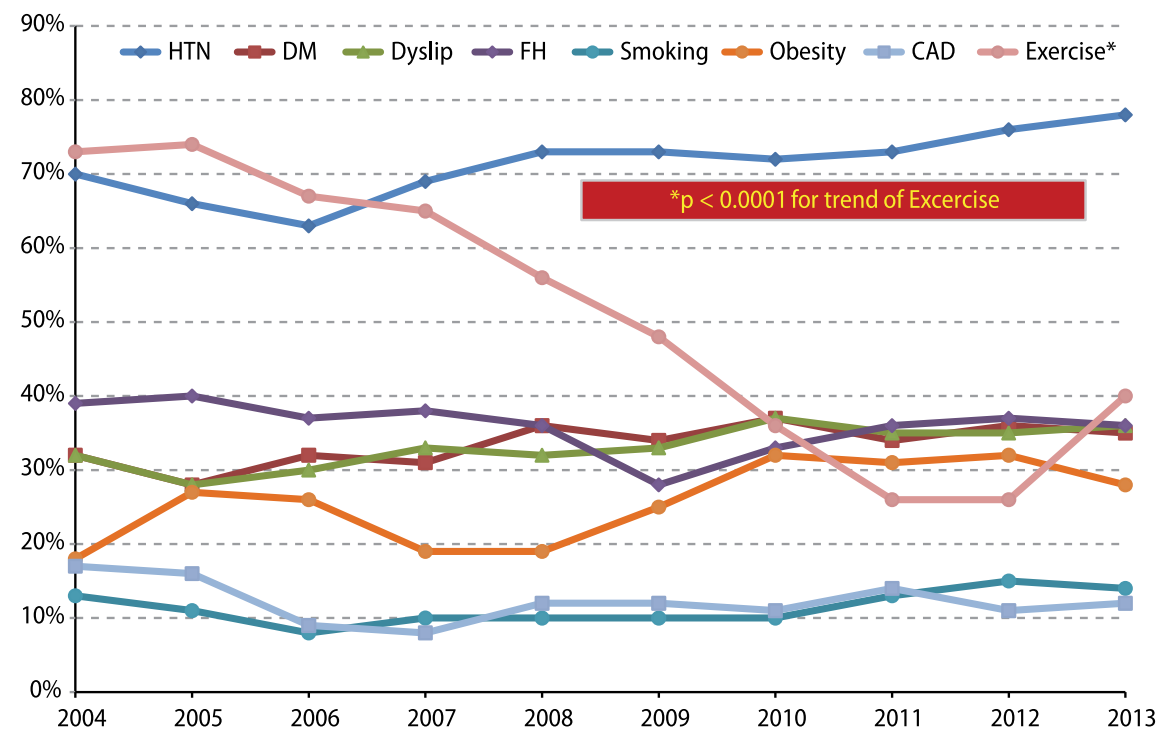

Figure 3. Demographical annual \%incidence trend from 2004 to 2013 for normal MPI

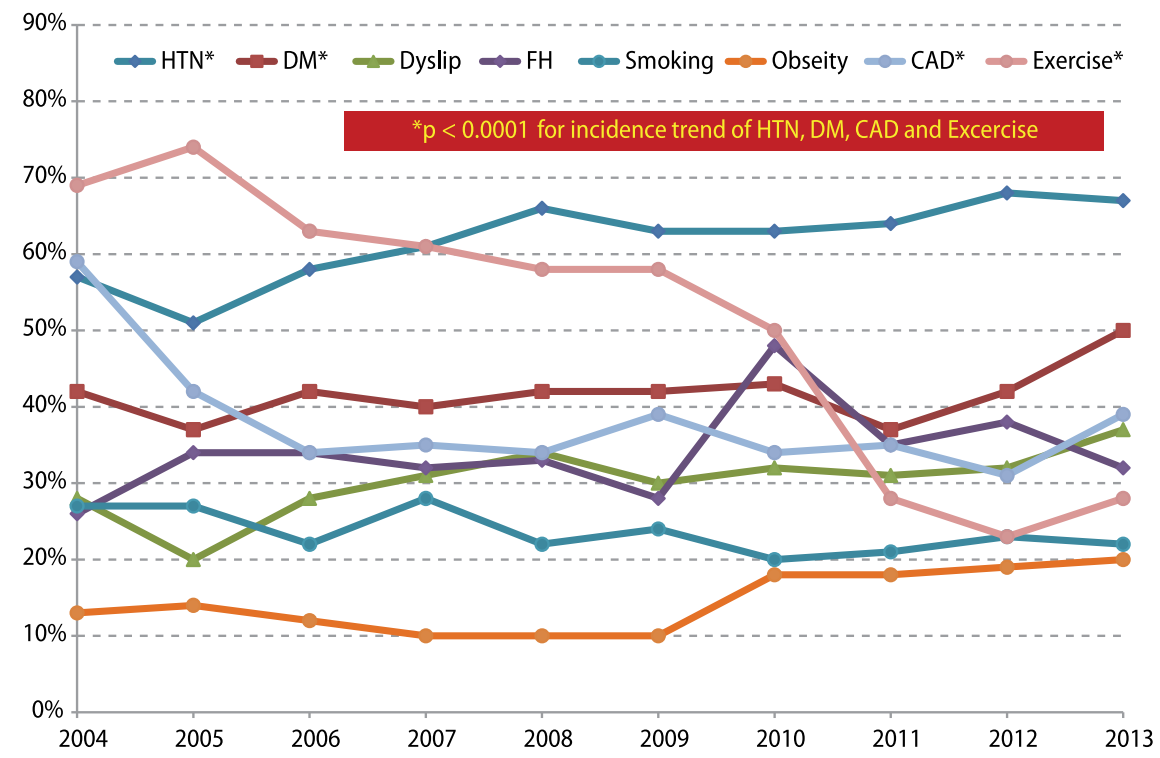

Figure 4. Demographical annual \%incidence trend from 2004 to 2013 for abnormal MPI

of stress, and a sedentary life style in studied population could be the prime reason for preference towards pharmacological (vasodilator) intervention over exercise as stress method. It is generally considered that MPI results obtained with vasodilators stress have shown good concordance with exercise stress [8, 9]. However, recent studies have shown lower negative predictive value (NPV) of a normal MPI done with vasodilator than with dynamic exercise $[10,11]$. We cannot exclude possible contribution of higher false negative results to the rising trend of normal MPIs in our cohort, as coronary angiography was not justified in these cases. Our results are in concordance with a study published by Rozanski et al. [3], although their study exhibited a very steep declining trend of abnormal MPIs from $40.9 \%$ to $8.7 \%$ in 20 years. The primary reason could be the larger sample size and longer study duration in their study.

Comparing patients with normal and abnormal MPIs on yearly basis, older age, male gender predominance, lower BMI, diabetes and smoking were found to have significant correlation with abnormal than normal MPIs. Except lower BMI, the remaining are well established risk factors for CAD and favor contribution towards an abnormal MPI. Reverse epidemiology and obesity paradox as discussed above may be the possible reasons for an unusual association between lower BMI and abnormal MPIs, which needs to be explored appropriately.

Interestingly in patients' cohort with a marginal rising trend of normal MPIs, only dynamic exercise did show an exaggerated declining trend while rest of known risk factors did not do exhibit any significant change in trend. The possible explanation could be the referral bias or possible higher false negative rate (lower NPV) of vasodilator stress MPI [12]. While marginal but significant declining trend of abnormal MPIs was found to be associated with significant rising trend of DM and HTN. This finding is in contradiction to previous studies where significant decline in abnormal MPIs was associated with reduction in risk factors [3]. One plausible 


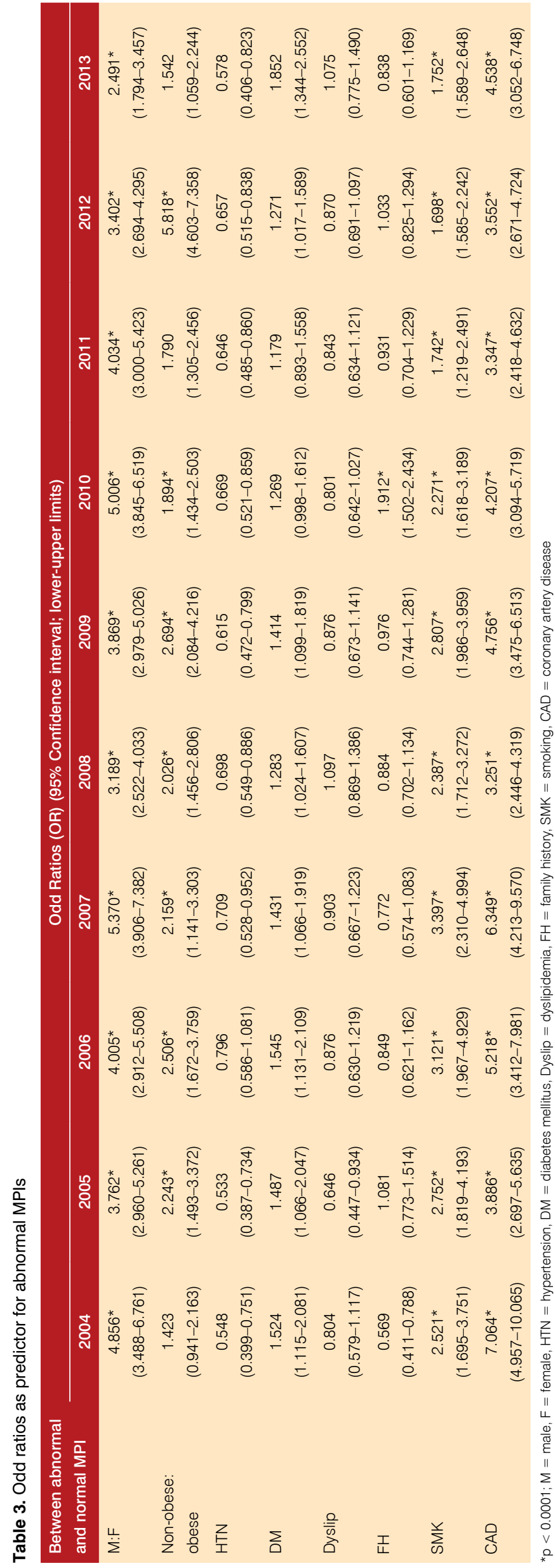

explanation for this finding could be the referral bias and subjecting more patients with DM and HTN (mixed population with or without symptoms for ischemia) for MPIs studies. Regarding asymptomatic diabetics, various prospective studies have shown a lower (6-22\%) prevalence of ischemia [13]. However, we have not segregated patients with or without ischemic symptoms and this is a limitation of the study. Another limitation of this study is lack of follow up of patients especially those with normal MPIs to find out NPV. The result of this study should be read carefully, as marginal but significant rise in normal MPI over a period 8.5 years has been associated with significantly higher trends of vasodilators stress which has lower NPV than exercise. We envisaged following these cases to ascertain the NPV of normal MPIs which will be shared in future.

We conclude that over the past 8.5 years, a marginal but significant decline in abnormal and a rise in normal MPIs trend have been observed. An exorbitant rise in use of vasodilator as method of stress has also been observed. We envisaged a follow-up study to ascertain lower NPV of vasodilator as a possible reason and till than results of this and other such studies must be read cautiously.

\section{References}

1. Go V, Bhatt MR, Hendel RC. The Diagnostic and Prognostic Value of ECG-Gated SPECT Myocardial Perfusion Imaging. J Nucl Med 2004; 45: 912-921.

2. Lloyd-Jones D, Adams RJ, Brown TM et al. Executive Summary: Heart Disease and Stroke Statistics - 2010 Update A Report From the American Heart Association. Circulation 2010; 121: 948-954.

3. Rozanski,A, Gransar H, Hayes SW et al. Temporal Trends in the Frequency of Inducible Myocardial Ischemia During Cardiac Stress Testing (1991 to 2009). J Am Coll Cardiol 2013; 61: 1054-1065

4. Abbas S, Kitchlew AR, Abbas S. Disease Burden of Ischemic Heart Disease in Pakistan and its Risk Factors. Ann Pak Inst Med Sci 2009; 5: 145-150.

5. Fang J, Wylie-Rosett J, Cohen HW, Kaplan RC, Alderman MH. Exercise, body mass index, caloric intake, and cardiovascular mortality. Am J Prev Med 2003; 25: 283-289.

6. Elhendy A, Schinkel AFL, Domburg RT et al. Prognostic Stratification of Obese Patients by Stress 99mTc-Tetrofosmin Myocardial Perfusion Imaging. J Nucl Med 2006; 47: 1302-1306.

7. Kang X, Shaw LJ, Hayes SW et al. Impact of Body Mass Index on Cardiac Mortality in Patients With Known or Suspected Coronary Artery Disease Undergoing Myocardial Perfusion Single-Photon Emission Computed Tomography. J Am Coll Cardiol 2006; 47: 1418-1426.

8. Levine MG, Ahlberg AW, Mann A et al. Comparison of exercise, dipyridamole, adenosine, and dobutamine stress with the use of Tc-99m tetrofosmin tomographic imaging. J Nucl Cardiol 1999; 6: 389-396.

9. Nishimura S, Mahmarian JJ, Boyce TM, Verani MC. Equivalence between adenosine and exercise thallium-201 myocardial tomography: a multicenter, prospective, crossover trial. J Am Coll Cardiol 1992; 20: 265-275.

10. Rozanski A, Gransar H, Hayes SW, Fiedman JD, Hachamovich R, Berman DS. Comparison of long-term mortality risk following normal exercise vs adenosine myocardial perfusion SPECT. J Nucl Cardiol 2010; 17: 999-1008.

11. Zaman M, Fatima N, Ishaq $M$ et al. Higher events rate in patients with a normal gated myocardial perfusion imaging with dipyridamole than exercise: "Run for reliability". Indian J Nucl Med 2012; 27: 72-77.

12. Navare SM, Mather JF, Shaw LJ, Fowler Ms, Heller GV. Comparison of risk stratification with pharmacological and exercise stress myocardial perfusion imaging: a meta-analysis. J Nucl Cardiol 2004; 11: 551-561.

13. Scholte AJHA. Cardiac risk assessment in asymptomatic diabetes: Combing different imaging modalities and surrogate markers? J Nucl Cardiol 2011; 18: 393-395 